\title{
Automated Mapping of Pre-Computed Module-Level Test Sequences to Processor Instructions *
}

\author{
Sankar Gurumurthy, Shobha Vasudevan and Jacob A. Abraham \\ Computer Engineering Research Center \\ University of Texas at Austin \\ \{sankar, shobha, jaa\}@ cerc.utexas.edu
}

\begin{abstract}
Executing instructions from the cache has been shown to improve the defect coverage of real chips. However, although the faults detected by such tests can be deteremined, there has been no technique to target test generation for an undetected fault. This paper presents a novel technique to map pre-computed test sequences at the module level of a processor, to sequences of instructions. The module level pre-computed test sequence is translated into a temporal logic property and the negation of the property is passed to a bounded model checker. The model checker produces a counter-example for the temporal logic property. This counter-example trace contains the instruction sequence that can be applied at the primary inputs to produce the pre-computed test sequence at the module inputs. This technique has no restrictions on the type of test sequences, so it can be used to map test sequences for any kind of fault to processor instructions. It can also be used in the design phase to produce validation tests.
\end{abstract}

\section{Introduction}

State-of-the-art VLSI designs present a major challenge to testing for defects after manufacture. Sequential ATPG techniques are typically inefficient for large, complex designs. In order to improve test quality, Design For Testability (DFT) techniques based on scan [6] and Built-InSelf-Test (BIST) [7] are employed. However, these techniques involve tradeoffs in power, performance and chip area. Moreover, as the processor geometries decrease, delay defects affect the functionality and performance of the processor. At-speed tests are necessary to detect these defects.

${ }^{*}$ This work was supported in part by Intel Corporation, and in part by Subcontract No.SA3271JB from UC Berkeley under prime Contract 2003-DT-660 from Microelectronics Advanced Research Corporation (MARCO)
The Murphy experiments [12] show that at-speed tests identify more defective chips than scan based tests for the same test vectors.

An example of at-speed processor testing in an industrial setting is FRITS [16]. FRITS showed the benefits of instructions executing from the cache for native mode self test [18]. These tests were composed of random instructions and helped to detect the defects that escaped the normal test flow. It is desirable to improve the fault coverage of the random tests by targeting hard-to-detect faults that escape detection by random instruction sequences.

In order to target hard-to-detect faults, it is beneficial to have knowledge about the instructions that can excite the faults. Although generic sequential ATPG tools are optimized using various techniques [1], [15], [17], they are not effective for large designs like processors. These ATPG tools can, however, be used effectively to generate test sequences for faults at the module level. These pre-computed test sequences ${ }^{1}$ can then be mapped to primary inputs. The input space of the processor is decided by its instruction set. Therefore, the pre-computed test sequences can be mapped to instruction sequences. Once the instruction sequence that produces a pre-computed test sequence is determined, it can be loaded into the processor cache and executed in native mode [18], thereby enabling at-speed testing. In addition, there will be no issues with power during test, because only legal instruction sequences are applied.

Mapping module-level pre-computed test sequences to instruction sequences is a challenging and as yet unsolved problem. Gate level descriptions of processors tend to be large and complex. Traditional ATPG operates at the gate level and is therefore ineffective for processors. Hierarchical techniques for test generation [19] are more efficient for large designs. However, they also do not solve the problem entirely. It is more promising to apply an ATPG-like algorithm at the Register Transfer Level (RT-Level), due to the

\footnotetext{
${ }^{1}$ We use the term pre-computed test sequences interchangeably with module level test sequences for faults within the module.
} 
inherent modularity, intuitiveness and relative simplicity of the RT-Level.

Murray et al. [14] deal with test generation for precomputed test sequences at RT-Level. However, their technique requires the design to be acyclic. Bhatia et al. [2] propose a solution that required the control and datapath to be separate. Lingappan et al. [10] represent the RT-Level design as algebraic decision diagrams (ADD) and use abstractions to represent the components of the ADD.

Generating instruction sequences for native mode testing has attracted considerable interest in the recent years. Kranitis et al. [8] presented a method to generate deterministic programs for self-testing of arithmetic modules. This method, however, requires knowledge of functionality of each block within the RT-Level implementation. Lai et al. [9] developed a behavior model of instructions which models the effect of each instruction on registers and major signal lines within the processor. This method involves enumerating all possible instruction pairs and examining every one of them. Corno et al. [5] use evolutionary techniques to direct the search process. Chen et al. [4] also deal with mapping of module level sequences to instructions. They extract module signals and map them to instruction templates using pre-defined mapping functions. However, their technique depends on the quality of the instruction templates and the mapping functions. Efficient instruction templates require in-depth knowledge of the processor design and the instruction set architecture. Significant manual intervention is required to get efficient mapping functions. Moreover their technique (like [8] and [5]) cannot guarantee mapping of a random test sequence.

We present a novel technique to map module level precomputed test sequences to instructions. Our technique works at the RT-Level and makes use of the hierarchy of the processor design. Our technique uses bounded model checking [3], rather than traditional ATPG algorithms, since the mapping problem can be written easily as a model checking problem. Bounded model checking is a formal verification technique that alleviates the state explosion problem of traditional model checkers by unrolling the model only into a specified bound. The bound is similar in concept to timeframes in ATPG. The verification result produced by BMC is valid within the bound specified.

Our approach to the problem is quite general. We do not try to separate the control and datapath of the processor, unlike [2]. Our technique operates on the actual RT-Level source code implementation of the design, as opposed to dealing with an abstraction as in [10]. We do not enumerate all pairs of instructions as in [9]. Unlike [8] we do not need to know the functionality of each block. Our technique can be used to target specific pre-computed test sequences. Our technique is generic and can be applied to any off-the-shelf processor. It is intended for use by the designer and requires minimal expertise.

Bounded model checking is used for both controllability and observability analysis of pre-computed test sequences. Controllability of a given test sequence is dealt with in the following way. We generate a Linear Temporal Logic (LTL) [11] safety property for controllability from a given precomputed test sequence. Cadence SMV's bounded model checker (BMC) [21] is then used to verify the generated property. We also constrain the BMC input space by providing it the instruction set of the processor. The property is written such that if the pre-computed test sequence can be generated through the instruction set, then BMC provides a counter-example for the property. We term the test sequences that can be generated through the instruction set as functionally feasible. The counter-example generated by BMC will contain a possible instruction sequence that can produce the given pre-computed test sequence. We compute a sufficient bound for BMC. If no counter-example is produced by BMC within this bound, it implies that the pre-computed test sequence cannot be produced by any instruction sequence. These are functionally infeasible precomputed test sequences because they are uncontrollable. Pre-computed test sequences are also functionally infeasible if they are not observable. Observability of the precomputed module level test sequences is handled in a similar manner, by applying BMC iteratively. Checking for controllability of pre-computed test sequences is fully automated.

We performed experiments on the OR1200, an open source RISC processor available from [20]. Test sequences were generated at the module level for faults which escaped detection from a long, random instruction sequence. These were our pre-computed test sequences. We demonstrate some of these pre-computed test sequences to be functionally infeasible. For the functionally feasible pre-computed test sequences, we show the instruction sequences that were mapped. These instruction sequences were also fault simulated at the chip level as a validity check. The main contributions of this paper are as follows.

1. We introduce a technique for mapping pre-computed test sequences to instructions, using bounded model checking.

2. Our technique works at the RTL source code level. The technique is therefore generic, and can be applied to off-the-shelf processors.

3. We provide a method to check the observability, as well as the functional feasibility of pre-computed test sequences using bounded model checking.

4. Our technique leverages the correctness of an existing formal verification engine for solving the instruction mapping problem. 
The rest of the paper is organized as follows. Section 2 describes the algorithm of the technique in detail. The implementation using OR1200 is discussed with suitable examples in Section 3. Section 4 gives the results of our experiments. The contributions of this paper and additional applications of the technique are discussed in detail in Section 5 .

\section{Instruction mapping of pre-computed test sequences}

Our technique maps pre-computed test sequences to instructions for processors. It is applied to RT-Level implementations of processor designs. We present the details of our technique in this section.

We use bounded model checking for the instruction mapping process. We write properties in LTL for BMC. Bounded model checking proves partial correctness of properties, i.e., if a counter-example is not generated within a given bound for a safety property, the property holds true within that bound. If the property does not hold (fails) within the given bound, then a counter-example is generated.

We apply bounded model checking to instruction mapping of pre-computed test sequences for every module of the processor's design. Pre-computed test sequences can be treated as primitives [14] that are available at the boundary (inputs) of an internal module. For every module of the processor, we translate a pre-computed test sequence into an LTL property. The property ties the signals to the corresponding values in the pre-computed test sequence. We negate this property and pass the resulting safety property through BMC. If a counter-example is produced, it implies that the signal values given in the pre-computed sequence can be generated by an instruction sequence of the processor.

The connection between the module inputs and the processor inputs (instruction sequence) is made due to the hierarchical operation of BMC. Figure 1 shows this operation pictorially. In Figure1, the solid arrows show the input/output dependencies between modules and the dashed arrows give the hierarchical flow of a counter-example. M3 is the module under test (MUT). The pre-computed test sequence at the inputs of M3 is converted into a LTL property and passed through BMC. The counter-example, if generated by BMC, contains the values of outputs ('o' in the figure) of M1 and M2. The counter-example also provides values of all the intermediate signals of M1 and M2 (as shown by dotted arrows) and the inputs ('i' in figure) of M1 and M2. Therefore, the counter-example provides signal values until the instruction ' $\mathrm{I}$ ', which is the input of the main module M0. We constrain the input space of BMC by providing it the instruction set of the processor under test. BMC is also

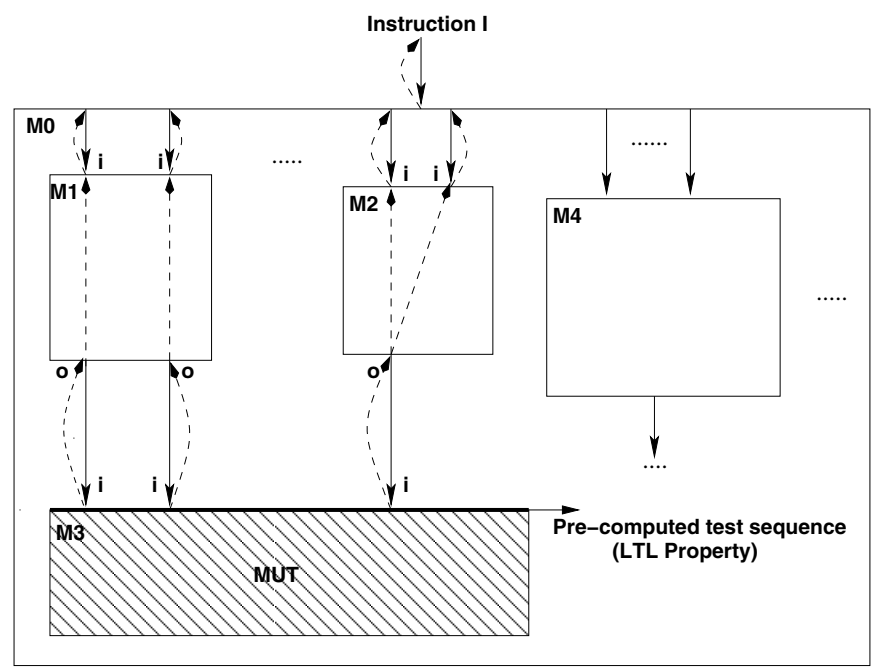

Figure 1. A hierarchical structure given to BMC. The dashed arrows give hierarchical flow of the counter-example and the solid arrows show the input/output dependencies between modules.

used for extracting observability constraints and checking the functional feasibility of pre-computed test sequences. A sufficient bound is computed and given to BMC for each stage of our method. The computation of the bound for the controllability stage is natural in the case of in-order pipelined processors with the external stalls disabled. In such a processor an instruction which enters the pipeline exits after $N$ cycles, where $N$ is the pipeline depth of the processor. Therefore, the effect of the instruction is felt for $N+1$ cycles if there is pipeline forwarding and for $N$ cycles if there is no forwarding. Hence, the bound should be more than the sum of the pipeline depth and the number of cycles in the pre-computed test sequence. If there are multicycle instructions, then the number of extra cycles taken by the longest instruction should also be added to get the bound. Since we do not force BMC to start from any particular state, no additional bound needs to be provided for initialization. The bound for observability stage is derived iteratively. On completion of controllability and observability, the instruction mapping is complete.

Figure 2 gives the flowchart for the technique. The shaded boxes in the flowchart are implemented using BMC. We start with a pre-computed test sequence and first analyze its controllability. In general, controllability deals with the possibility of generating a desired value for an internal signal. We check if the given pre-computed test sequence can be generated through the instructions in the instruction set of the processor. We need to define controllability property 

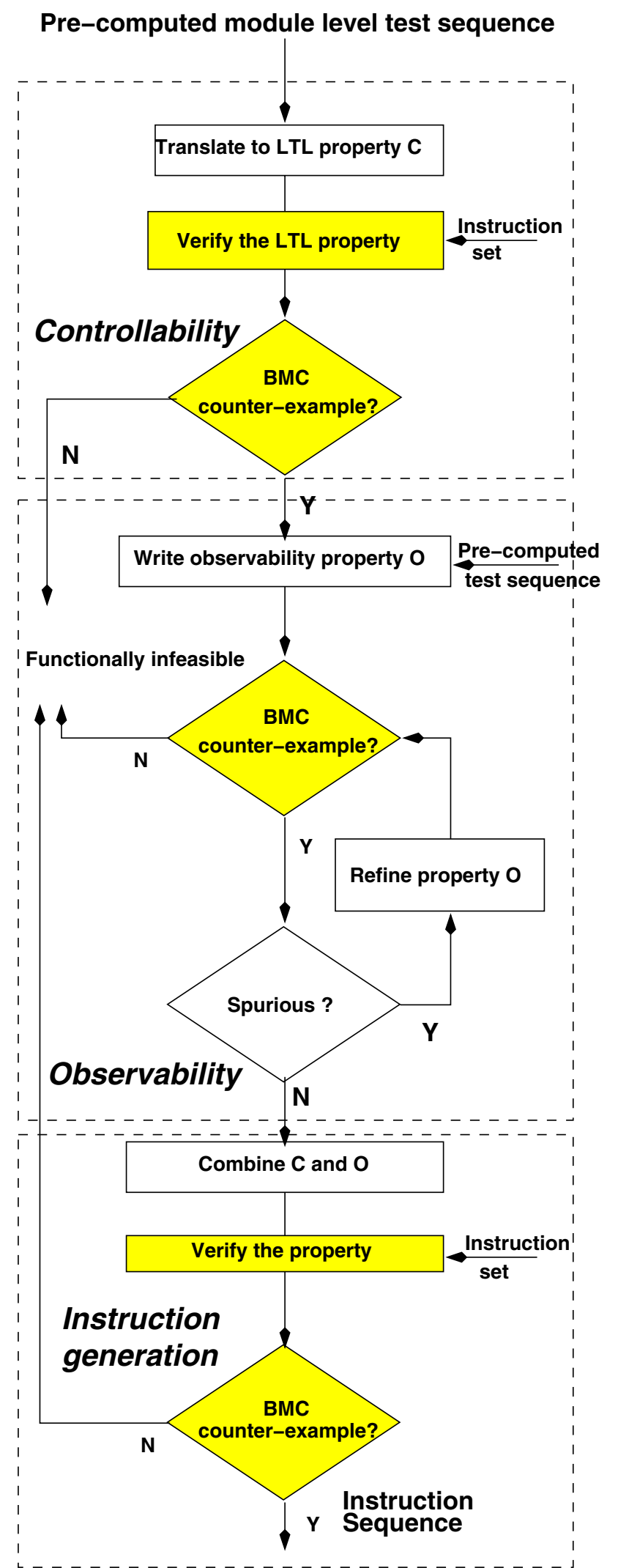

Figure 2. Flowchart for the instruction mapping of pre-computed test sequences. The shaded boxes are implemented using BMC

\begin{tabular}{|l|lll|}
\hline time & $a$ & $b$ & $c$ \\
\hline 0 & 1 & 0 & $\mathrm{x}$ \\
1 & 1 & 1 & $\mathrm{x}$ \\
2 & $\mathrm{x}$ & $\mathrm{x}$ & 1 \\
\hline
\end{tabular}

Table 1. An example test sequence

$C$ for this purpose.

The controllability property $C$ is defined such that, if it fails, the counter-example generated by BMC will contain the desired instruction sequence at the inputs of the processor.

For example, the test sequence given in Table 1 is first translated to the LTL property:

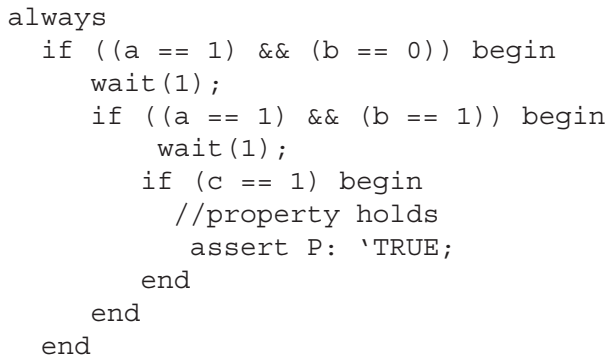

This property is then negated and built into an assertion. This assertion is the desired controllability property $C$. For the example test sequence, $C$ is:

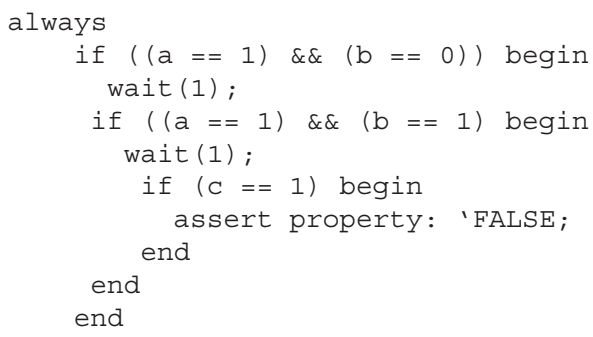

The property states that $a, b$ and $c$ can never have the values given in the test sequence. The values in times 0 , 1 and 2 are modeled using the next state operator in LTL, which is represented by the wait statement in Verilog.

We pass the controllability property $C$ to $\mathrm{BMC}$ along with the processor's RT-level source code. BMC is also given the instruction set of the processor under test. The bound for BMC is calculated using the method described earlier.

BMC checks for the existence of a counter-example for the stated property within the given bound. If no such counter-example exists, then the pre-computed test sequence is functionally infeasible, since it is not controllable. The technique proceeds only if there is a counter-example.

The pre-computed test sequence also identifies the module outputs that need to be propagated to observable points to make it observable. Therefore, in the observability stage of the technique, we extract constraints needed to propagate 
these module outputs to an observable point of the processor. The observable points of a processor include primary outputs, memory, register file and all other user accessible points of the processor.

Since we use BMC for observability, we define an LTL property for observability. The observability property $O$ is defined such that if it fails, the counter-example generated by BMC will contain the constraints necessary for propagation of the module outputs. Therefore, observability property $O$ states that a change in a module output signal should not cause a change in any of the observable points of the processor. This property is of the form:

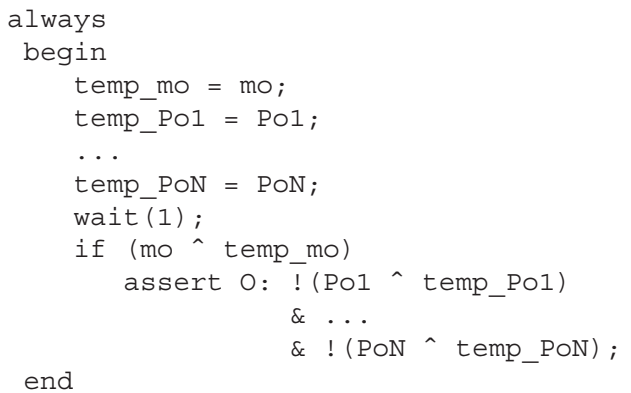

where mo is the module output that needs to be propagated and $\mathrm{PO} 1, \mathrm{PO} 2, \ldots$ PoN are the observable points.

The property states that a change in the value of mo in the next state does not imply that eventually one of the observable points will change their value. A change is modeled by using an xor operator between the signal value in the current state and the next state.

Property $O$ is passed to BMC. If no counter-example is generated, then the test sequence is functionally infeasible since it is not observable. However, existence of a counterexample does not necessarily prove that module output is observable. For example, consider the Verilog model given below:

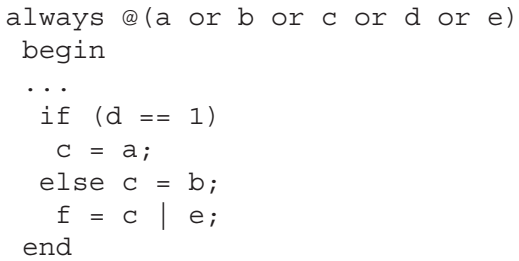

Let a be the module output which needs to be propagated and $f$ be the only observable point. The initial observability property $O$ would then be:

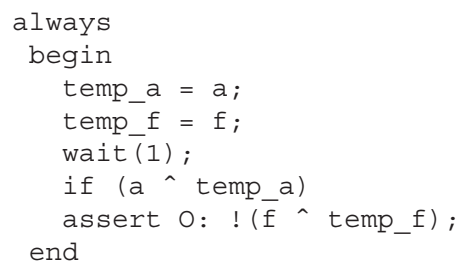

It is possible then that BMC generates the counterexample:

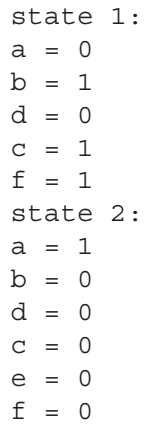

The counter-example shows a change in the value of a and a change in the value of $f$, which disproves the property $O$. However, a has not actually been propagated. We term such a counter-example as spurious. A counter-example is spurious if it disproves the observability property, although it does not guarantee propagation of a pre-computed test sequence. Therefore, the property $O$ has to be refined, to generate the correct counterexample, and thereby, the correct constraints. For the given example, $O$ is refined by adding the constraint that the value of d should be set. Hence, the refined property $O$ is:

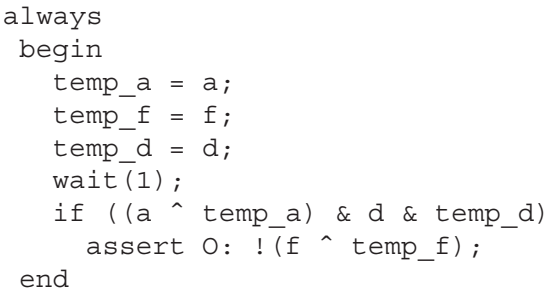

The counter-example generated for this property gives all the necessary constraints for observability. In general, the property $O$ is iteratively refined by adding constraints based on spurious counter-examples, until a counter-example that actually models the propagation is found. The bound can also be iteratively changed after starting with pipeline depth as the initial bound. Note that the instruction set is not given as a constraint to BMC while checking for observability. There is no need to constrain the inputs to the instruction set, Adding instruction set information to the property places the unnecessary constraint of finding an instruction sequence which will cause a change in the module output. This change in module outputs is caused only when there is a fault, and does not depend on instructions.

The extracted observability property $O$, is combined with the controllability property $C$ using simple conjunctions, and passed to BMC. At this stage, BMC is also given the instruction set as a constraint. The bound for this stage is determined by the sum of the number of cycles needed for controllability and observability constraints. If BMC produces a counter-example then the instruction sequence is extracted from the counter-example. This is the required instruction sequence generated by our technique. 


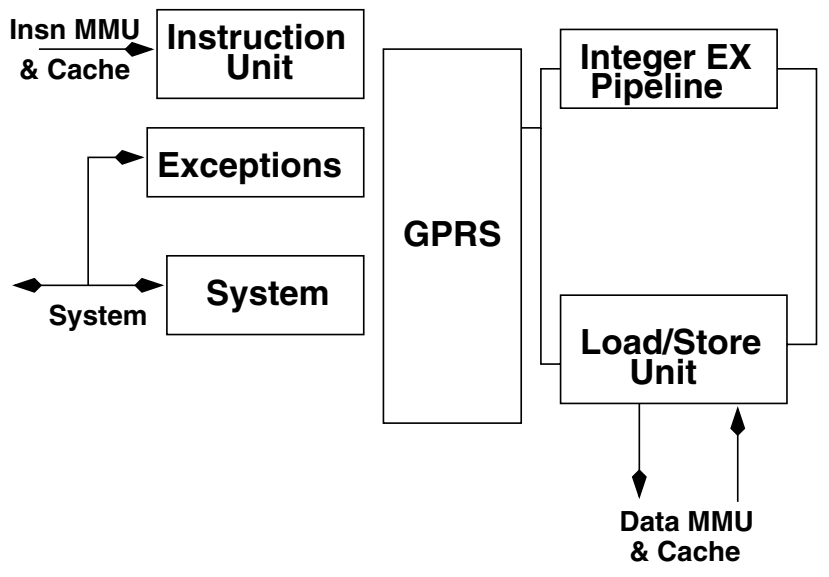

Figure 3. OR1200 CPU's block diagram

It is possible that BMC does not find a counter-example. This might be because of one of two possibilities. The first possibility is that the controllability and observability properties contradict each other, i.e, the signal values obtained in the counter-examples for $C$ and $O$ have conflicting values. In that case, BMC eliminates the pre-computed test sequence. The second possibility is that the observability constraints do not coincide with the possible instruction sequences. This is possible, because we extract observability constraints from BMC without providing it the instruction set as a constraint. If no counter-example is generated, the pre-computed test sequence is declared as functionally infeasible.

\section{Mapping pre-computed test sequences to instructions for OR1200}

\subsection{OR1200}

The OR1200 is a publicly available processor design. The source code in Verilog RTL of OR1200 is available from [22]. The specification manual can also be found at [22].

The OR1200 is a 32-bit scalar RISC processor, with a Harvard microarchitecture, 5 stage integer pipeline, virtual memory support (MMU) and basic DSP capabilities. The CPU of the OR1200 has an instruction unit that implements the basic instruction pipeline. There are 32 general purpose registers (GPRs) of 32-bits each in OR1200. The load store unit handles all the transfer between GPRs and the internal bus of CPU. There is also an exception handling unit which implements a uniform procedure for all exceptions. The integer execution unit of OR1200 executes most integer instructions in one cycle. The basic block diagram for OR1200 is given in Figure 3.

\subsection{Generating pre-computed test sequences for OR1200}

To the best of our knowledge, there are no pre-computed test sequences available for OR1200. Therefore, we need to generate pre-computed test sequences at the module level. In order to select faults in a module to target for the process, We wrote a random instruction sequence which has 36750 instructions. The OR1200 was fault simulated with this instruction sequence, and the fault coverage saturated around $68 \%$ for stuck- at faults ${ }^{2}$. We split the undetected faults into separate lists depending on the module to which they belong. A commercially available ATPG tool was used to generate pre-computed test sequences for each of these faults. These pre-computed test sequences were then fault simulated at the module level to add information about module outputs that would change their values, in case of a fault. These test sequences were used as the pre-computed test sequences.

\subsection{Generating LTL properties from pre- computed test sequences}

OR1200 has various external inputs that can cause a stall in the pipeline. This helps the processor communicate correctly with the environment. However, we need to ensure that the counter-examples generated by BMC do not stall the processor due to these external inputs. Similarly, we also need to ensure that the counterexamples do not reset the processor. We state the above constraints as part of our controllability property. The instruction set information is added to the property, to constrain its input space.

Setting the constraints for the properties is a one time operation. These constraints remain the same for all precomputed test sequences. Each pre-computed test sequence is translated to its corresponding LTL property, and the constraints are added to it. For example, for the test sequence (for the module operandmuxes) given in Table 2:

\begin{tabular}{|c|c|c|c|c|}
\hline time & id_freeze & ex_freeze & sel_b[1] & sel_b[0] \\
\hline 0 & 0 & 0 & $\mathrm{X}$ & $\mathrm{X}$ \\
1 & $\mathrm{X}$ & 0 & 1 & 0 \\
\hline
\end{tabular}

\section{Table 2. A pre-computed test sequence for OR1200}

the controllability property, is given in Figure 4:

We have defined IF_INSN_LEGAL such that it is set if the instruction fetched in the current cycle is legal (part of the instruction set of the OR1200). As shown in the property

\footnotetext{
${ }^{2}$ There is no necessity to limit the fault model to stuck-at faults. We use stuck-at faults to illustrate our technique.
} 


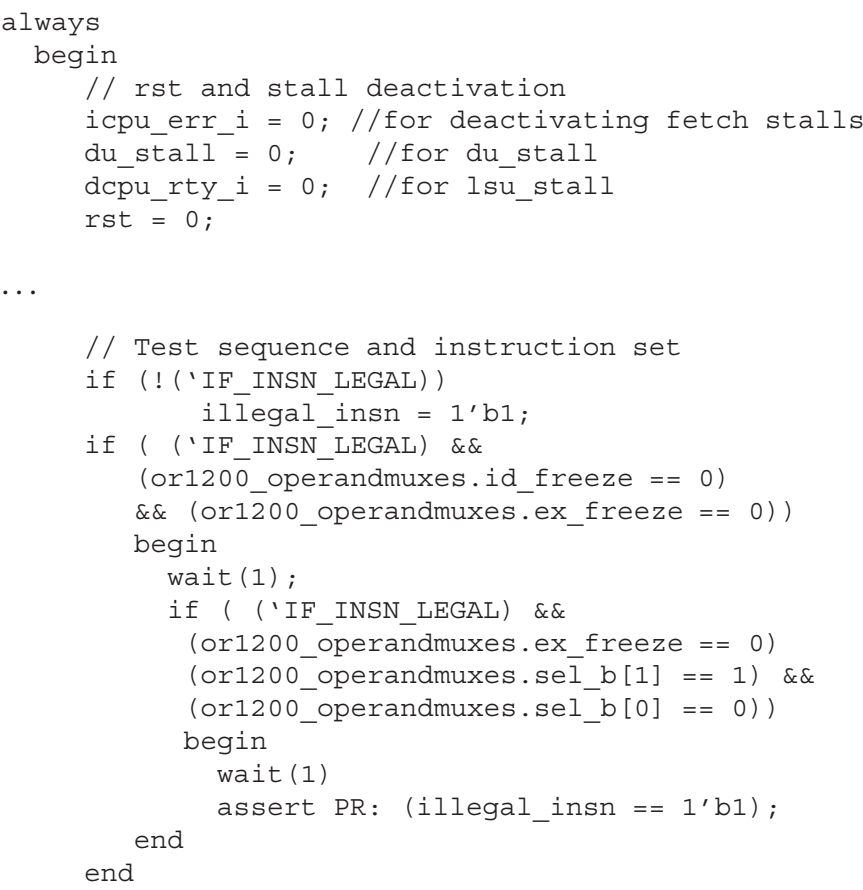

\section{Figure 4. OR1200 controllability property ex-} ample

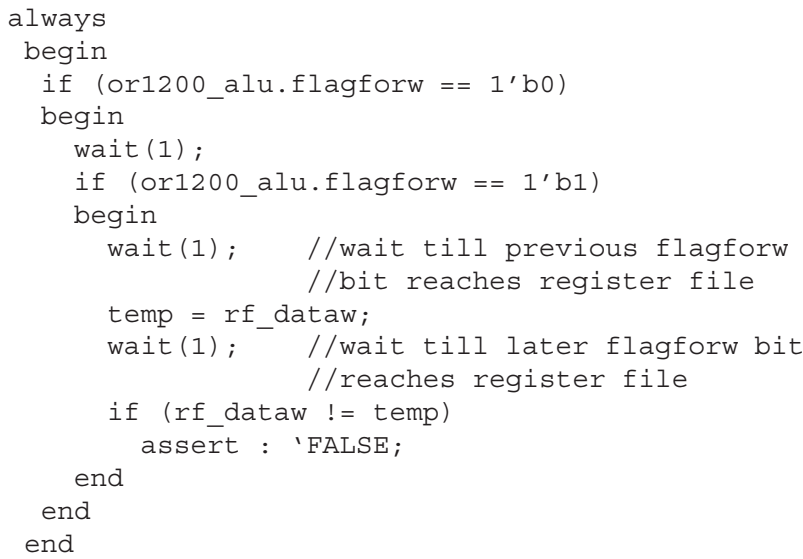

Figure 5. Property for observability

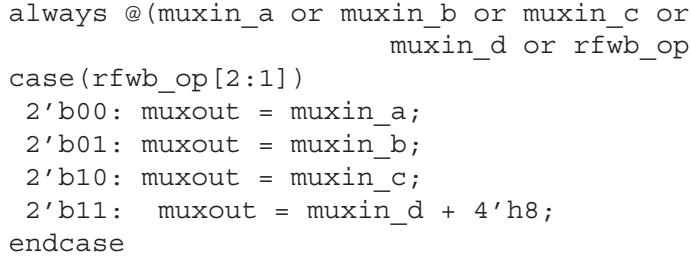

Figure 6. Verilog code snapshot

of $r f w b \_o p$ set to 00 . In this case flagforw is blocked from propagating further. Therefore, the property has to be refined to add the constraint that the most significant bits of rfwb_op need to be tied to the value 10. A counterexample that is not spurious is obtained after adding this constraint.

Therefore, the property for observability is iteratively refined to achieve the desired results. However, once the constraints are extracted for propagation of all the outputs of a module, all the pre-computed sequences for that module can be propagated using the same constraints.

\subsection{Instruction generation}

We have automated the generation of the controllability property as well as checking its functional feasibility. All the pre-computed test sequences generated as described in Subsection 3.1 were checked for controllability. As shown in Section 4 many of those pre-computed test sequences are functionally infeasible. Once a pre-computed test sequence passes the controllability test, an observability property is defined for it and signal values for output propagation are extracted. The refined observability property is combined with the controllability property to get the overall property of instruction mapping. The counter-example generated by BMC for this property contains an instruction sequence that would generate the pre-computed test sequence. 


\begin{tabular}{|l|c|c|}
\hline Module & $\begin{array}{c}\text { No. of } \\
\text { pre-computed sequences }\end{array}$ & $\begin{array}{c}\text { Uncontrollable } \\
\text { Test sequences }\end{array}$ \\
\hline alu & 2812 & 14 \\
genpc & 2964 & 2345 \\
sprs & 4626 & 3243 \\
wbmux & 1053 & 33 \\
lsu & 1401 & 87 \\
freeze & 85 & 23 \\
ctrl & 1620 & 437 \\
except & 5965 & 269 \\
operandmuxes & 421 & 35 \\
if & 1688 & 279 \\
\hline
\end{tabular}

\begin{tabular}{|l|c|c|}
\hline Module & $\begin{array}{c}\text { No. of pre-computed } \\
\text { test sequences }\end{array}$ & No. of Instructions \\
\hline alu & 9 & 18 \\
ctrl & 5 & 23 \\
operandmuxes & 5 & 19 \\
\hline
\end{tabular}

Table 4. Instruction mapping for each module

\section{Table 3. Result for feasibility check on pre- computed test sequences}

If no counter-example is found then the pre-computed test sequence was declared as infeasible.

\section{Experimental results}

Experiments were performed on OR1200 processor [20]. A long pseudo-random instruction sequence containing 36750 instructions was generated for the OR1200 CPU core. These 36750 instructions were generated by randomly varying the data operands for the instructions of OR 1200. The OR1200 CPU core was fault graded for all possible stuck-at faults for the random instruction sequence using a commercially available tool. The fault coverage saturated around $68 \%$. The list of faults that were left undetected formed the base list. We applied our technique on this base list. We thereby simulated hard-to detect faults which are the principal target of the technique.

We split the base list based on modules. We generated the pre-computed test sequences by the method described in Subsection 3.2. We checked functional feasibility for all the pre-computed test sequences that were generated. Table 3 shows the results for the functional feasibility check of all the pre-computed test sequences.

The first column in Table 3 gives the name of each mod$u_{1}^{3}$. The number of pre-computed test sequences for each module (which is same as the number of faults in each module that were undetected by the random instruction sequence) is given in the second column. The number of test sequences that were functionally infeasible is given in third column.

Out of the pre-computed test sequences designated as functionally feasible, we picked some sequences at ran-

\footnotetext{
${ }^{3}$ The names of the modules have been shortened due to space constraints. The actual names of the modules can be obtained from the source code.
}

dom and we generated an instruction sequence for each of them. We kept track of observability constraints that were extracted for each pre-computed test sequence and re-used them if the same module output had to be made observable for some other pre-computed test sequence.

BMC was allowed to start from any state allowing registers to contain any value at the beginning of the instruction sequence generated by BMC. Therefore, we added an initialization sequence which loaded those values into registers. OR1200 has a load-store architecture. Therefore, it is easier to initialize the registers to desired values. Table 4 shows the results of our instruction mapping for the example modules. The results have been shown on control (ctrl) and datapath (alu, operandmuxes) modules.

The first column in Table 4 gives the name of the module. The second column gives the number of pre-computed test sequences for which the instructions were generated. The total number of instructions that were generated for the selected pre-computed test sequences is shown in the third column. We fault simulated the instruction sequences at chip level and checked if they detected the fault corresponding to the pre-computed test sequence.

We also found that the technique lends itself well to prioritizing instructions. If the user wants to assign higher priority to certain instructions, then instruction set input to $\mathrm{BMC}$ can be constrained to those instructions. This can be useful if the user wants to avoid instructions like load and store which are multicycle operations.

Table 5 gives some examples of instruction sequences that were generated for some of the pre-computed test sequences from Table 4. The first column gives the module name. The second column gives the location of the fault within the corresponding module. The type of fault is indicated in the third column. The instruction sequence generated by our mapping technique is shown in the fourth column. These instructions belong to the OR1200's instruction set architecture. (available from [22]). The fifth column gives the number of other faults from the base list that were detected by this instruction sequence.

It can be observed from the table that the instruction sequence generated for a specific fault also detected many other faults from the base list. The instruction sequence shown for $c t r l$ module detected more than 2000 other faults 


\begin{tabular}{|c|c|c|c|c|}
\hline Module & Pin path & Fault type & $\begin{array}{l}\text { Instruction } \\
\text { Sequence }\end{array}$ & $\begin{array}{c}\text { No. of other faults } \\
\text { detected from base list }\end{array}$ \\
\hline alu & /U2723/C & s-a-1 & $\begin{array}{l}\text { 1.ori } \mathrm{r} 31, \mathrm{r} 0,0 \mathrm{xffff} \\
\text { 1.cust5 }\end{array}$ & 441 \\
\hline ctrl & /U1091/A & s-a-1 & $\begin{array}{l}\text { 1.ori r31, r0, 0x4 } \\
\text { l.ori r30, r0, 0x2 } \\
\text { 1.nop } \\
\text { 1.sw 0x0(r31), r31 } \\
\text { 1.addc r31, r30, r31 } \\
\text { 1.mfspr r22, r1, 0xf }\end{array}$ & 2424 \\
\hline operandmuxes & /U680/C & $\mathrm{s}-\mathrm{a}-0$ & $\begin{array}{l}\text { l.andi } \mathrm{r} 1, \mathrm{r} 1,0 x f 813 \\
\text { l.movhi } \mathrm{r} 0,0 \mathrm{xf} 813 \\
\text { 1.addi } \mathrm{r} 0, \mathrm{r} 1,0 x f 813 \\
\text { 1.sw 0x13(r1), r31 } \\
\text { 1.movhi } 0,0 x f c f 3\end{array}$ & 463 \\
\hline
\end{tabular}

Table 5. Some example instruction sequences that were generated

from the base list. This is because the instruction sequence contained an instruction (l.mfspr) which operated on the special purpose register. This instruction was difficult to generate in the random instruction sequence. However, our technique generated an instruction sequence containing this instruction. We also found that 1.cust5 targeted most of the faults in the alu module. This was because 1.cust5 instruction does not give complete access to its operands making it is difficult to load it with appropriate values using random instruction sequences.

\section{Discussions and Conclusions}

We have shown a new technique for instruction mapping of pre-computed test sequences. We have used bounded model checking in our technique. In the technique's flow, BMC can be replaced with a traditional model checker and the technique would still be correct. Mishra et al. [13] provide a way for targeting interesting cases in the pipeline for validation using a traditional model checker. However, they use an abstraction of the processor. Therefore, it is not possible to target any random case since it might not exist in the abstraction. The blowup associated with using traditional model checkers on large designs makes it inefficient to use them without abstracting the design. Bounded model checking has been proposed as a viable alternative to traditional model checking. It provides a partial notion of correctness within the given bound. Although bounded model checking does not guarantee correctness outside the bound, a counterexample obtained during bounded model checking, is definitely valid within that bound. Since our technique translates the pre-computed test sequences into safety properties, a counterexample implies that the safety property does not hold within the specified bound. The no- tion of pipeline depth in processors provides a natural way to compute a sufficient bound for BMC. This makes it possible to check for functional infeasibility of pre-computed test sequences.

A major advantage of the technique is that we leverage an existing formal verification engine for solving a problem, that has traditionally been solved by ATPG engines. The existing formal verification engine ensures the automatic and correct operation of the justification like algorithm. Since we add the properties at the RT-Level, any bounded model checking tool can be used for our method. We used SMV because it was an easily available and robust tool. We can easily substitute it with an engine which works at RT-Level or uses word-level reasoning.

The main overhead of the technique could be viewed as writing the LTL properties. We have automated the generation of controllability properties. There is no manual intervention required to check the controllability of a precomputed test sequence. Manual intervention is required only when the observability property has to be refined. The use of techniques like observe only scan out chains [16] could avoid that requirement. Another method to circumvent writing the observability properties, is to generate precomputed sequences whose observability has already been computed.

The proposed technique can be integrated with other test generation frameworks like FRITS [16]. Our technique can be used to target the faults that escape detection by those tools.

As mentioned in [14], pre-computed test sequences are not specific to any particular fault model. Therefore, our technique can be used to detect various kinds of faults like stuck-at faults, delay faults etc. In fact a fault model is not even necessary, since the pre-computed sequence could be 
meant for exciting a particular state etc. This is very useful while debugging the design of a processor.

Automation of the functional infeasibility check is a desirable feature. This avoids time spent in trying to map infeasible sequences. The functional infeasibility check helps targeting hard-to-detect faults because it is more probable that the pre-computed test sequences generated at the module level for these faults are functionally infeasible.

Our technique is generic, requires minimal manual intervention, and uses the power of existing formal verification techniques to solve the instruction mapping problem. The technique has been shown to work with an off-the-shelf processor, and is targeted to be used by the RT-Level designer.

This technique could be used for generating alternate test sequences if the pre-computed test sequences are declared functionally infeasible. We can feed back the information regarding feasibility of the original pre-computed sequence to the ATPG tool to get different sequences from the ATPG tool.

\section{References}

[1] R. Bencivenga, T. J. Chakraborty, and S.Davidson. The architecture of the gentest sequential test generator. In Proceedings of the Custom Integrated Circuits Conference, pages 17.1.1-17.1.4, May 1991.

[2] S. Bhatia and N. K. Jha. Integration of hierarchical test generation with behavioral synthesis of controller and data path circuits. IEEE Transactions on VLSI Systems, pages 608619, Dec 1998.

[3] A. Biere, A. Cimatti, E. M. Clarke, and Y. Zhu. Symbolic model checking without bdds. In Proceedings of the 5th International Conference on Tools and Algorithms for Construction and Analysis of Systems, pages 193-207. SpringerVerlag, 1999.

[4] L. Chen, S. Ravi, A. Raghunathan, and S. Dey. A scalable software-based self-test methodology for programmable processors. In Proceedings of the 40th Design Automation Conference, pages 548-553, June 2003.

[5] F. Corno, G. Cumani, M. S. Reorda, and G. Squillero. Fully automatic test program generation for microprocessor cores. In Proceedings of the conference on Design, Automation and Test in Europe, pages 1006- 1011. IEEE Computer Society, 2003.

[6] E. B. Eichelberger and T. W. Williams. A logic design structure for LSI testability. In Proceedings of the 14th Design Automation Conference, pages 462-468, June 1977.

[7] G. Hetherington, T. Fryars, N. Tamarapalli, M. Kassab, A. Hassan, and J. Rajski. Logic BIST for large industrial designs: real issues and case studies. In Proceedings of the International Test Conference, pages 358-367, Sep 1999.

[8] N. Kranitis, A. Paschalis, D. Gizopoulos, and Y. Zorian. Effective software self-test methodology for processor cores. In Proceedings of the conference on Design, automation and test in Europe, pages 592-597. IEEE Computer Society, 2002.
[9] W.-C. Lai, A. Krstic, and K.-T. Cheng. Test program synthesis for path delay faults in microprocessor cores. In Proceedings of the International Test Conference, pages 1080-1089. IEEE Computer Society, 2000.

[10] L. Lingappan, S. Ravi, and N. K. Jha. Test generation for non-separable RTL controller-datapath circuits using a satisfiability based approach. In Proceedings of the 21st International Conference on Computer Design, pages 187-193, Oct 2003.

[11] Z. Manna and A. Pnueli. The temporal logic of reactive and concurrent systems. Springer-Verlag New York, Inc., 1992.

[12] E. J. McCluskey and C. W. Tseng. Stuck-Fault Tests vs. Actual Defects. In Proceedings of the International Test Conference, pages 336-343, Oct 2000.

[13] P. Mishra and N. Dutt. Automatic functional test program generation for pipelined processors using model checking. In Proceedings of the IEEE International High Level Design Validation and Test Workshop, pages 99-103, Oct 2002.

[14] B. T. Murray and J. P. Hayes. Hierarchical test generation using pre-computed tests for modules. IEEE Transcations on Computer-Aided Design of Integrated Circuits and Systems, pages 594-603, June 1990.

[15] T. M. Niermann and J. H. Patel. HITEC: A test generation package for sequential circuits. In Proceedings of the European Conference on Design Automation, pages 214-218, Feb 1991.

[16] P. Parvathala, K. Maneparambil, and W. Lindsay. FRITS a microprocessor functional BIST method. In Proceedings of the International Test Conference, pages 590-598, Oct 2002.

[17] M. H. Schulz and E. Auth. ESSENTIAL: An efficient selflearning test pattern generation algorithm for sequential circuits. In Proceedings of the International Test Conference, pages 28-37, Aug 1989.

[18] J. Shen and J. A. Abraham. Native mode functional test generation for processors with applications to self test and design validation. In Proceedings of the International Test Conference, pages 990-999, Oct 1998.

[19] R. S. Tupuri and J. A. Abraham. A Novel Functional Test Generation Method for Processors Using Commercial ATPG. In Proceedings of the International Test Conference, pages 743-752. IEEE Computer Society, Nov 1997.

[20] OR1200 RISC processor. http://www.opencores.org.

[21] BMC engine of Symbolic Model Verifier. http://wwwcad.eecs.berkeley.edu/ $/ \mathrm{kenm} \mathrm{cmil} / \mathrm{smv} /$.

[22] OR1200 documentation and source code.

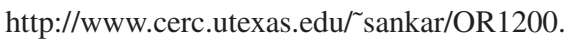

